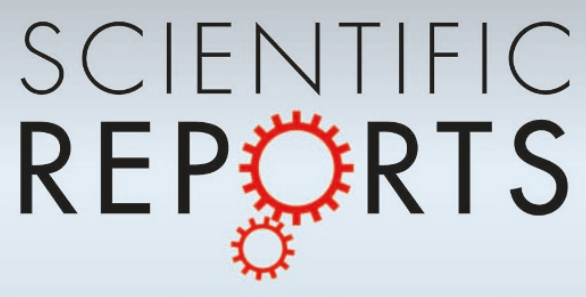

OPEN

SUBJECT AREAS:

METAMATERIALS

SUB-WAVELENGTH OPTICS

Received

8 December 2014

Accepted

16 March 2015

Published

5 June 2015

Correspondence and requests for materials should be addressed to

D.P.T. (dptsai@phys. ntu.edu.tw)

\title{
Plasmon coupling in vertical split-ring resonator metamolecules
}

\author{
Pin Chieh Wu', Wei-Lun Hsu', Wei Ting Chen', Yao-Wei Huang' ', Chun Yen Liao', Ai Qun Liu², \\ Nikolay I. Zheludev ${ }^{3,4}$, Greg Sun ${ }^{5} \&$ Din Ping Tsai ${ }^{1,6}$
}

\begin{abstract}
'Department of Physics, National Taiwan University, Taipei 10617, Taiwan, ${ }^{2}$ School of Electrical and Electronic Engineering, Nanyang Technological University, Singapore 639798, Singapore, ${ }^{3}$ Optoelectronics Research Centre and Centre for Photonic Metamaterials, University of Southampton, Southampton SO17 1BJ, UK, ${ }^{4} \mathrm{TPI}$ and Centre for Disruptive Photonic Technologies, Nanyang Technological University, Singapore 637371, Singapore, ${ }^{5}$ Department of Engineering, University of Massachusetts Boston, Boston, MA 02125 , U.S.A., ${ }^{5}$ Research Center for Applied Sciences, Academia Sinica, Taipei 11529 , Taiwan.
\end{abstract}

The past decade has seen a number of interesting designs proposed and implemented to generate artificial magnetism at optical frequencies using plasmonic metamaterials, but owing to the planar configurations of typically fabricated metamolecules that make up the metamaterials, the magnetic response is mainly driven by the electric field of the incident electromagnetic wave. We recently fabricated vertical split-ring resonators (VSRRs) which behave as magnetic metamolecules sensitive to both incident electric and magnetic fields with stronger induced magnetic dipole moment upon excitation in comparison to planar SRRs. The fabrication technique enabled us to study the plasmon coupling between VSRRs that stand up side by side where the coupling strength can be precisely controlled by varying the gap in between. The resulting wide tuning range of these resonance modes offers the possibility of developing frequency selective functional devices such as sensors and filters based on plasmon coupling with high sensitivity.

$\mathrm{P}$ lasmonic metamaterials composed of artificial sub-wavelength structures typically involving metal have gained tremendous interest during the past decade because of their extraordinary optical properties and potential applications ${ }^{1-5}$. These properties and applications of the metamaterials are intrinsically connected to the localized surface plasmon (SP) resonances (LSPR) arising from the collective oscillations of free electrons which induce strong electromagnetic fields adjacent to the artificial sub-wavelength metallic elements (referred to here as metamolecules) in the metamaterials $s^{6,7}$. Properties of metamaterials can be readily tailored by engineering their constituent metamolecules composed of subwavelength metal structures ${ }^{8}$. For instance, a metamolecule constructed with a pair of closely spaced plasmonic elements exhibits rather different optical response than those made of isolated ones ${ }^{9}$. The plasmonic coupling of metamolecules has been explored to achieve a number of applications, such as the Fano resonance ${ }^{10,11}$, toroidal dipolar response ${ }^{12,13}$, Rabi splitting ${ }^{14,15}$ and biosensors ${ }^{16}$. While these promising applications of metamaterials continue to extend beyond the reach of any conventional media, one cannot help notice that most of them are driven by the electric field of an incident electromagnetic wave $^{17}$. It is nevertheless desirable to expand the optical properties of these metamaterials to include their responses to magnetic field as well. Magnetic coupling through mutual inductive effects has been studied in in-plane coupled split-ring resonators (SRRs) ${ }^{18-22}$, but the dipoles were still excited by the incident electric field in experiment. The fact that a majority of previous studies have mainly focused on the plasmonic properties in the metamaterials that are mostly derived from the dipole response to the electric field of the incident wave acting upon these metamaterials is the direct consequence of significant technical challenges in the fabrication of metamaterials because they are far more easily constructed with planar sub-wavelength elements on substrates $^{23-25}$, and their magnetic dipole moments driven only by the electric field of incident electromagnetic wave are always perpendicular to the magnetic field of a normal incident wave, resulting in a weak interaction with the magnetic field ${ }^{26-28}$. Attempts have been made to address this issue with the use of multilayer metamaterials but the fabrication techniques are still challenging ${ }^{29-32}$. While the oblique incidence also allows for the magnetic response to be observed to a certain degree ${ }^{33,34}$, such an effect can be further enhanced with the fabrication of vertical splitring resonator (VSRR) structures in which the metamolecules stand up vertically, leading to their magnetic dipoles that can not only be excited by the electric field, but also by the magnetic field directly under normal incidence ${ }^{35-37}$. 
In this work, using a recently developed high precision alignment technique $^{38}$, we have fabricated VSRRs which allowed us to study how incident electromagnetic fields interact with these VSRRs and to reveal the plasmon coupling between closely spaced VSRRs in dimer structures (metamolecules). We first numerically compare the magnetic plasmon excitation between isolated SRRs that are in either planar or vertical configuration. We then fabricate and measure spectral transmittance of isolated VSRRs to identify their magnetic resonance. Taking advantage of the flexibility in arranging VSRRs that stand up side by side where the coupling strength of their magnetic dipoles can be tuned far more efficiently with their spacing, we have observed electric and magnetic plasmon coupling of two VSRRs of different dimensions resulting in a range of resonance shift. The vertical configuration enable more densely packed metamolecules for enhanced plasmonic properties.

\section{Results}

We have conducted numerical simulation using COMSOL to establish the comparison between single isolated planar and VSRR metamolecules of equal dimensions (base length $L=195 \mathrm{~nm}$ ) as shown in Figs. 1a and 1c, respectively, under the excitation of a normal incident wave with its electric field polarized along the SRR gap ( $\mathrm{x}$-axis). In this configuration, planar SRRs are driven by the incident electric field only because the incident magnetic field is perpendicular to their magnetic dipoles which get induced only because of the bianisotropy ${ }^{39,40}$, the VSRRs, on the other hands, are excited by both electric and magnetic components of the incident wave and the effect of bianisotropy includes excitations of electric and magnetic dipoles by magnetic and electric fields, respectively. Considering gold SRRs placed on a glass (BK7) substrate, we have simulated the magnetic response of both SRRs. For planar SRRs, the magnetic response is induced by the oscillating electric current in the SRR due to its interaction with the incident electric field and a distribution of magnetic energy density is present within the SRR opening between the prongs (Fig. 1b). In comparison, the VSRR structure has a clear advantage in that it couples directly with not only the electric field but also the magnetic field under normal illumination. Our simulation result indicates that stronger magnetic energy density can indeed be obtained under the same dimensions and illumination condition (Fig. 1d).

Inspired by the above simulation results, we proceed to fabricate VSRR structures with two different sizes as shown in Fig. 2 (right). The geometries for the two different structures are identical to the SRR dimensions used in simulation except the base length $L$. One sample has shorter base length of $170 \mathrm{~nm}$ while the other $220 \mathrm{~nm}$. The reason for us to study these VSRRs of two different sizes is to establish the baseline for our next step in investigating the plasmon coupling between two closely spaced VSRRs of the same two sizes. The periodical lattice spacing in both samples has been chosen to be $500 \mathrm{~nm}$ to avoid coupling with its neighbors so that these VSRRs can be treated as being isolated. We have performed transmittance measurement on the two samples and the results are shown in Fig. 2 (left). There is a pronounced resonance dip for each isolated VSRR around $1200-\mathrm{nm}$ wavelength which is the so-called LC resonance also referred to as the magnetic plasmon resonance because of the participation of magnetic dipole in the plasmon oscillation ${ }^{41}$. The resonance difference between the two isolated VSRRs of different dimensions is $\Delta \omega \approx 20 \mathrm{THz}$ in the absence of plasmon coupling between them. The deeper transmittane dip observed from the lager VSRRs is the result of their greater area coverage density over the substrate relative to their smaller counterparts.

We next investigate the resonance tuning of coupled VSRRs by fabricating a series of dimer samples with different spacing as shown schematically in Fig. 3a with the expectation to reveal the strong a

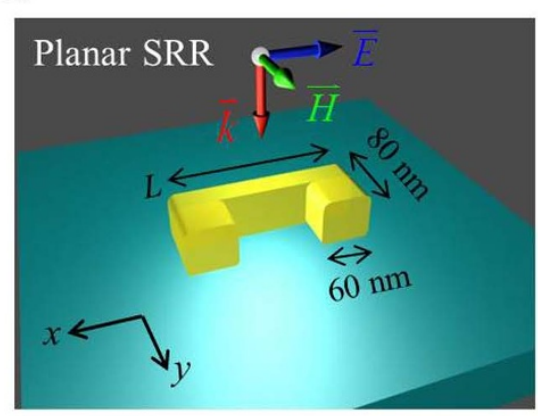

c

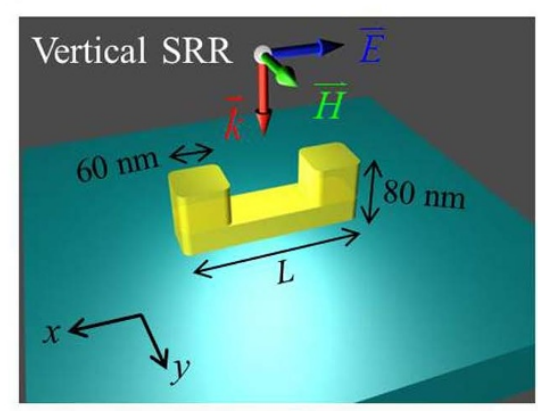

b

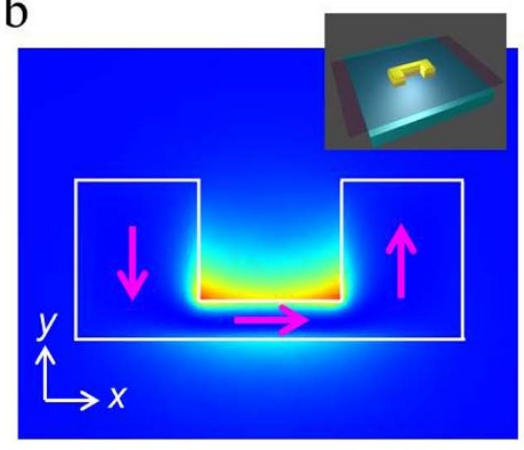

d

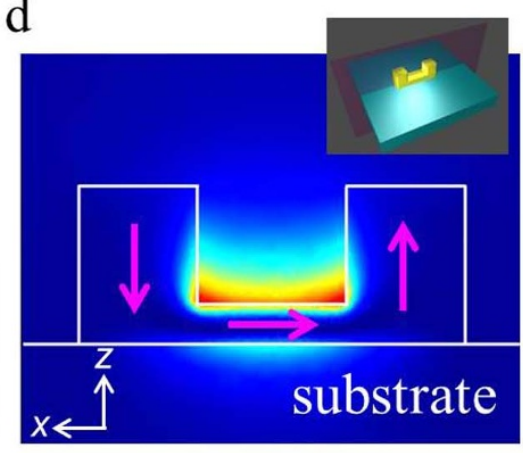

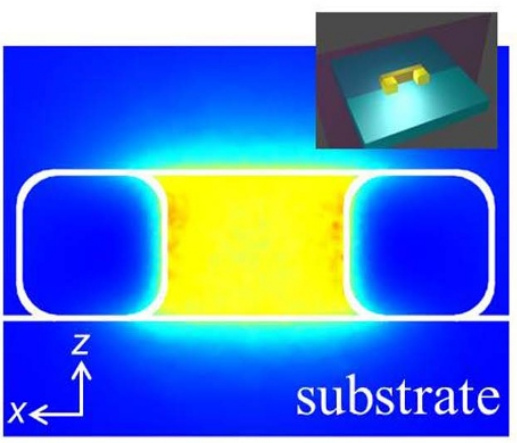

Max

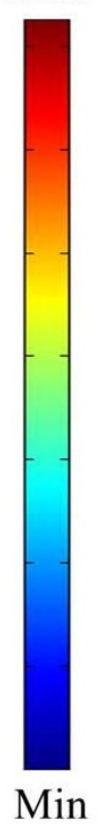

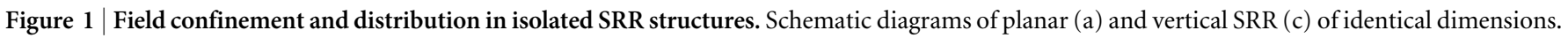

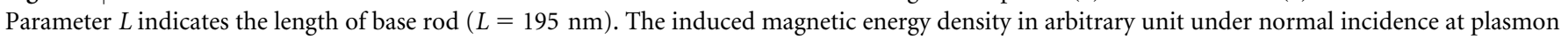
resonance is shown in two perpendicular planes for comparison. Observation planes are shown as insets in (b) and (d). The side views (left) in (b)

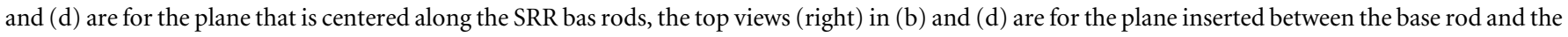
two prongs. Arrows in magenta indicate the direction of the induced surface current. 


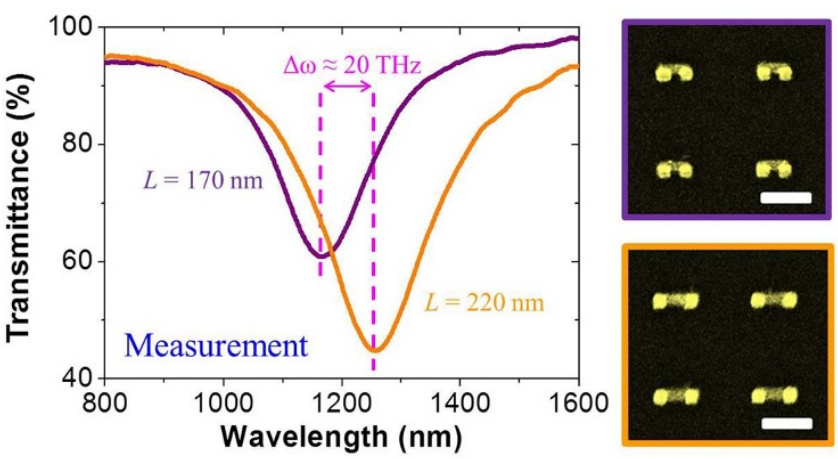

Figure 2 Electromagnetic response of isolated VSRR. Experimental transmittance spectra for isolated VSRR with $500 \mathrm{~nm}$ period in $\mathrm{x}$ and $\mathrm{y}$ directions on a glass (BK7) substrate. The purple and orange spectral lines correspond to the isolated VSRR with $L=170 \mathrm{~nm}$ and $220 \mathrm{~nm}$, respectively. The resonance difference is about $20 \mathrm{THz}$ in frequency indicated by the magenta dashed lines. The images on right represent the top view SEM pictures of the corresponding structures. Scale bars: $250 \mathrm{~nm}$.

magnetic dipole coupling in vertical dimers because VSRRs can be placed much closer to each other. The two coupled VSRRs have the same geometries except different base lengths of $L_{1}=170 \mathrm{~nm}$ and $L_{2}=220 \mathrm{~nm}$ (the resonance position of each when isolated is shown in Fig. 2). As shown in Fig. 3a they are placed in parallel along $\mathrm{x}$-axis with their centers aligned on $y$-axis. Figure $3 b$ shows the SEM images (oblique views) of the gold VSRR dimer sample with 50-nm gap separation fabricated on a glass (BK7) substrate. The inset in Fig. $3 b$ is an enlarged perspective view of four VSRR dimers with their two prongs sitting precisely on the two ends of the base rod.

Four VSRR dimer samples with gap separations $G$ of $40,50,70$ and $90 \mathrm{~nm}$ are fabricated and measured. All samples have the same lattice constant of $P=500 \mathrm{~nm}$ in both $\mathrm{x}$ and $\mathrm{y}$ directions between each dimer unit cell (metamolecules) to avoid coupling between VSRRs from neighboring unit cell. Figure 4a represents the transmittance spectra simulated at four different gap separations between the two VSRRs where two transmittance dips emerge. The measurement (Fig. 4b) of these VSRR arrays reveals similar resonance features in reasonably good agreement with the simulation. The difference between the measurement and simulation is due to the VSRR size variation and roughness of fabricated samples that deviate from the exact dimensions and boundary condition of perfectly smooth structures used in the simulation. The two transmittance dips are clearly associated with the magnetic plasmon modes that originate from the two VSRRs of different dimensions. As the separation between the two VSRRs reduces, the coupling between them becomes stronger, shifting the two resonances further apart as revealed from the simulation and measurement in Figs. $4 \mathrm{a}$ and 4b, respectively.

\section{Discussion}

Plasmon hybridization theory $\mathrm{y}^{42}$ has been proposed to reveal the origin of plasmon resonances of complex metal nanostructures as interactions between constituent elements much like the coupling between two closely spaced quantum structures where electron wavefunctions overlap. This theory has been proven successful in predicting and analyzing optical responses of assemblies of metal nanoparticles of various shapes including dimers among others ${ }^{43,44}$. The plasmon hybridization that has been reported so far primarily originates from interactions of electric and magnetic dipoles and higher-order multi-pole oscillations of individual nanoparticles that make up a complex nanostructure ${ }^{10,45}$. The VSRR dimer structures reported here offer a perfect venue to explore enhanced magnetic interaction between individual nanostructures that also influences the optical response of a composite metal structure. Indeed the VSRRs have much stronger magnetic coupling than those planar
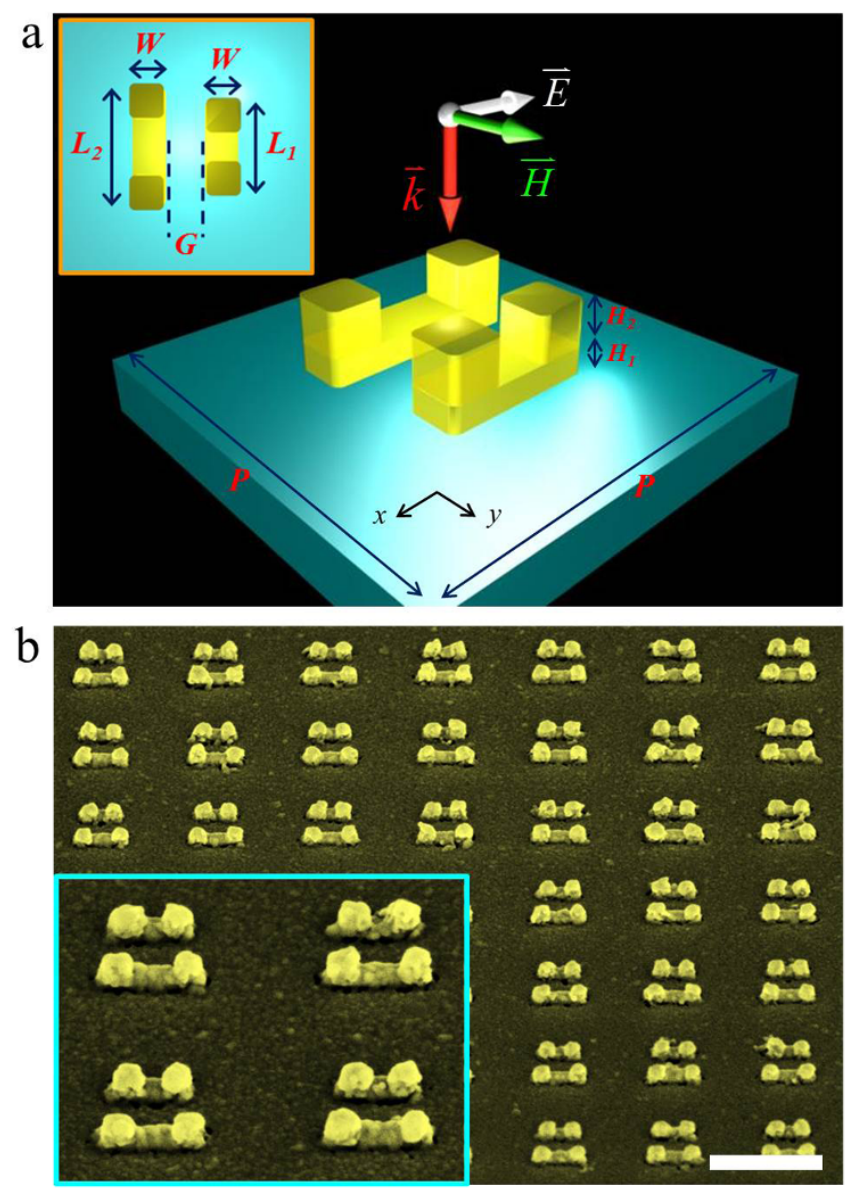

Figure 3 Geometry of VSRR dimer. (a) Schematic diagrams of VSRR dimer unit cell with designed parameters: $L_{1}=170 \mathrm{~nm}, L_{2}=220 \mathrm{~nm}$, $H_{1}=20 \mathrm{~nm}, H_{2}=60 \mathrm{~nm}, W=60 \mathrm{~nm}$ and $P=500 \mathrm{~nm}$. A parameter $G$ is introduced for the gap separation between VSRRs. (b) The $45^{\circ}$ SEM micrograph with the zoom-in view (inset) for the sample with $G=50 \mathrm{~nm}$. Scale bar: $500 \mathrm{~nm}$.

ones placed next to each other, and their coupling strength can be controlled by their spatial separation $G$. In the hybridization picture, each VSRR supports a dipole oscillation with its own plasmon resonance frequency at $\omega_{a}$ or $\omega_{b}$ depending on the VSRR dimensions, when two VSRRs are brought closer in the configuration shown in Fig. 3a their electric dipoles transversely couple to each other while the magnetic ones interact longitudinally, both contributing to the hybridization of resonance modes in the metamolecules that shifts the positions of original magnetic resonances $\omega_{a}$ and $\omega_{b}$ supported by the isolated VSRRs. It can be seen from the simulation result of the induced surface current distribution of the VSRR dimers that are separated by $G=50 \mathrm{~nm}$ under normal illumination in Fig. $4 \mathrm{c}$ that two resonance modes emerge from the coupled VSRRs, one associated with parallel induced electric currents in the two constituent VSRRs that enhances both electric and magnetic dipole moments, and the other with reduced moments from anti-parallel currents. The dominance of the electric coupling dictates that the "bonding" mode has out-of-phase electric dipoles, resulting in outof-phase magnetic dipole moment oscillation as well (marked as $\omega_{a-b}$ for their out-of-phase characteristic), while the "anti-bonding" mode (marked as $\omega_{a+b}$ ) has in-phase electric and magnetic dipoles. Since the two VSRRs have different dimensions, we have $\omega_{a} \neq \omega_{b}$, and if we assume $\omega_{a}<\omega_{b}$ the result of hybridization is to yield "bonding" and "anti-bonding" modes with their mode resonances separated further apart according to $\omega_{a-b}<\omega_{a}<\omega_{b}<\omega_{a+b}$. It is interesting to point out that the resonance at the longer wavelength 
a

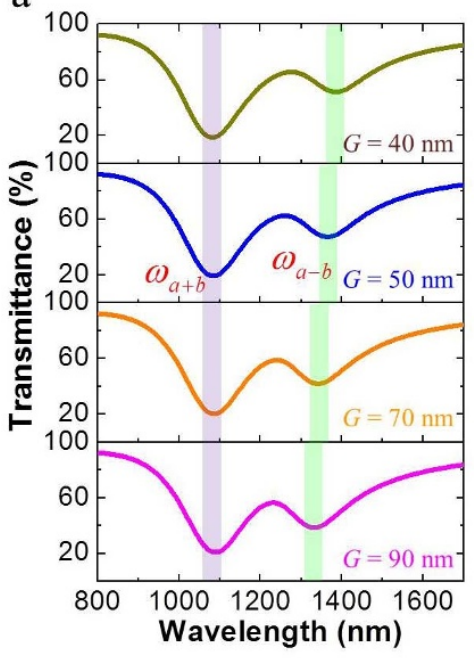

b

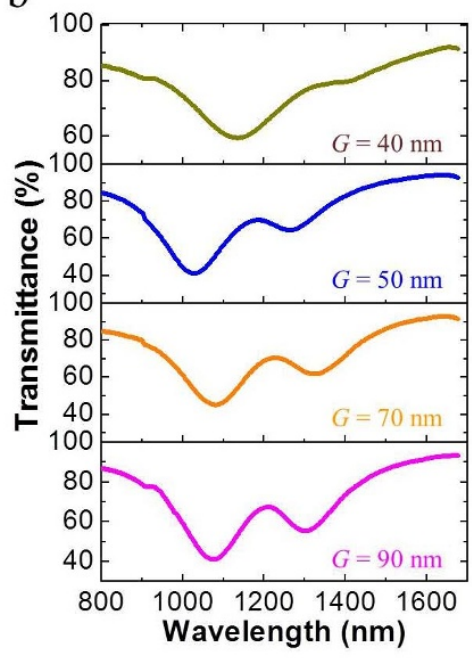

c

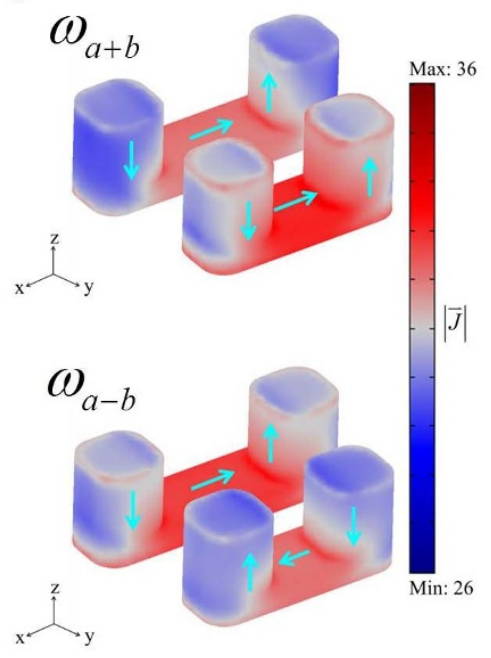

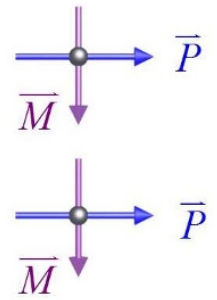
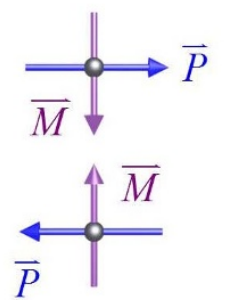

Figure 4 Electromagnetic spectra of VSRR dimers. (a) Simulation and (b) experimental transmittance spectra for VSRR dimers with various gaps. The colored shade highlights the position for each resonance mode. (c) Surface current density (in arbitrary unit and logarithmic scale) of the VSRR dimer with $G=50 \mathrm{~nm}$ under normal illumination at respective resonance wavelength $\omega_{a+b}$ and $\omega_{a-b}$ as indicated in (a). Arrows present the direction of induced surface current. Right: directions of induced electric and magnetic dipoles.

(marked $\omega_{\mathrm{a}-\mathrm{b}}$ ) appears to be weaker as the gap separation becomes smaller, because this "bonding" mode originated from the two opposing electric and magnetic dipoles in the dimer as shown in Fig. $4 \mathrm{c}$ interacts weakly with the incident field. As a consequence, the resonance feature in the transmittance spectra at the shorter wavelength is always stronger than the one at the longer wavelength as shown in Figs. $4 \mathrm{a}$ and $4 \mathrm{~b}$. This plasmon hybridization of two unequal VSRRs shifts the plasmon resonances much like the coupling between two interacting semiconductor quantum dots (QDs) of different sizes. While each QD supports a confined state with a different energy, the result of coupling because of their electron wavefunction overlap is that the two confined states are pushed further apart. The amount of energy shifting reflects the coupling strength which depends on the geometries of the two QDs and is particularly sensitive to their spatial separation. We have also observed the similar behavior in the coupled VSRRs by systematically varying the separation $G$ within a SRR dimer. Figure 5 shows the simulation and measurement of resonance frequency separation $\Delta \omega$ $=\omega_{a+b}-\omega_{a-b}$ of the "anti-bonding" and "bonding" for the four samples with VSRR spacing from 40 to $90 \mathrm{~nm}$ under normal illumination. The coupled resonance separation $\Delta \omega$ is consistently

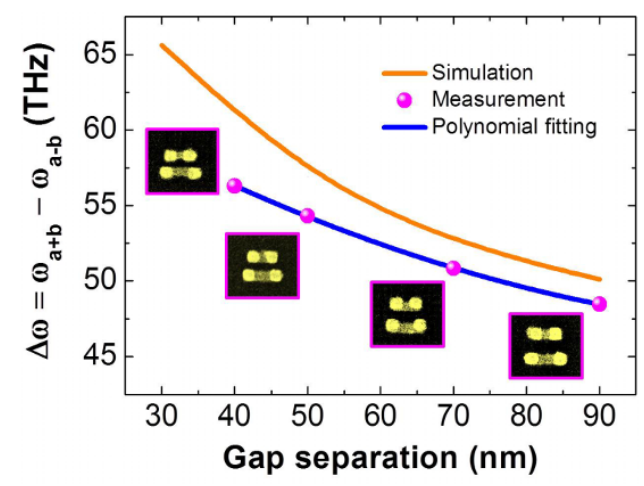

Figure 5 | Bonding and anti-bonding splitting vs. the gap in VSRR dimer. Orange line and magenta dots correspond to simulation and measurement results, respectively. Blue line: a second-order polynomial fitting for experimental results. Insets represent the corresponding SEM image with gap sizes $G=40,50,70$ and $90 \mathrm{~nm}$ between SRRs (left to right). greater than the resonance frequency difference $(\sim 20 \mathrm{THz})$ obtained from the transmittance measurement of the isolated VSRRs of same two different sizes shown in Fig. 2. As the spacing $G$ between the VSRRs reduces, $\Delta \omega$ increases rapidly with the decreasing $G$.

To summarize, we have fabricated a series of metamolecules consisting of either isolated VSRRs or their coupled dimers with different SRR spacing using e-beam lithography with high precision alignment technique. These VSRR metamolecules have the advantage of direct coupling to both the electric and magnetic components of the normal incident wave in comparison to their planar counterpart that only interacts with the electric field, resulting in stronger magnetic response. By conducting simulation and measurement of the optical transmittance, we have observed hybridization of magnetic plasmon modes associated with constituent VSRRs in metamolecules where bonding and anti-bonding modes emerged. We have found that the energy separation between the bonding and anti-bonding modes in metamolecules depends strongly on the gap separation in VSRR dimers. The tuning capability enabled by the magnetic plasmon mode coupling can be explored for developing frequency selective functional devices.

\section{Methods}

Fabrication of VSRRs. VSRR structures with different feature sizes are fabricated using electron beam lithography with high precision alignment technology. A $200 \mathrm{~nm}$-thick $495 \mathrm{~K}$ PMMA (polymethyl methacrylate) layer was spin-coated at $4000 \mathrm{rpm}$ on cover glass and then baked for $3 \mathrm{~min}$ at $180^{\circ} \mathrm{C}$. The conductive polymer Espacer is then spin-coated at $1500 \mathrm{rpm}$ over the PMMA layer to avoid the charging problem during the e-beam exposure process. An ELS-7000 electron beam lithography system (Elionix Inc., Tokyo, Japan) is used for exposure with $100 \mathrm{keV}$ acceleration voltage and 30-pA current. The position of the VSRR base rod was defined on the PMMA resist relative to the two 100-nm-thick gold cross alignment marks which were first fabricated on the substrate for precise alignment during ebeam exposure process. After exposure, the sample was rinsed with de-ionized water to remove Espacer, then developed in solution of methyl isobutyl ketone (MIBK) and isopropyl alcohol (IPA) of MIBK:IPA = 1:3 for 60 seconds, rinsed again, this time with IPA, for 20 seconds, and blow-dried with nitrogen gas. Once the development of the resist was completed, a gold film with designed thickness was thermally deposited on the sample, and the un-patterned regions were removed using a lift-off process. Subsequently, the two VSRR prongs were fabricated in a similar fashion by the second e-beam exposure and lift-off process. The area of each fabricated structure is $75 \times 75 \mu \mathrm{m}^{2}$ on a cover glass substrate.

Optical measurement and simulation. The spectra were measured by a selfassembled micro-spectrometer, and an inverted Olympus microscope IX-70 (10× IR 
objective with numerical aperture $\mathrm{NA}=0.3$, long working distance condenser with $\mathrm{NA}=0.3$, visible to near-infrared polarizer U68-750 from Edmoud Optics and $100 \mathrm{~W}$ halogen light source) equipped with two spectrometer (BTC111E for $\lambda=$ $400 \mathrm{~nm}$ to $\lambda=1000 \mathrm{~nm}$ with $\sim 0.5 \mathrm{~nm}$ resolution and BTC261E for $\lambda=900 \mathrm{~nm}$ to $\lambda=1700 \mathrm{~nm}$ with $\sim 5 \mathrm{~nm}$ resolution) from B\&W Tek, Inc. All transmittance spectra were normalized by an un-patterned region of the cover glass substrate.

FEM Simulation. All simulation results were performed with the commercial software COMSOL Multiphysics by solving 3D Maxwell equations. Both isolated and coupled VSRR dimers are simulated with periodic boundary conditions under $\mathrm{x}$ polarized light illumination. The refractive index of cover glass substrate is fixed at 1.51. The permittivity of gold in the near infrared regime is described by the DrudeLorentz model with plasmon frequency $\omega_{p}=8.997 \mathrm{eV}$ and damping constant $\Gamma_{p}=$ $0.14 \mathrm{eV}$, which is two times larger than that of the bulk value because of the surface scattering and grain effects.

1. Zheludev, N. I. \& Kivshar, Y. S. From metamaterials to metadevices. Nat. Mater. 11, 917-924 (2012).

2. Lal, S., Link, S. \& Halas, N. J. Nano-optics from sensing to waveguiding. Nat. Photon. 1, 641-648 (2007).

3. Sun, G., Khurgin, J. B. \& Yang, C. C. Impact of high-order surface plasmon modes of metal nanoparticles on enhancement of optical emission. Appl. Phys. Lett. 95, 171103 (2009).

4. Chen, W. T. et al. High-efficiency broadband meta-hologram with polarizationcontrolled dual images. Nano Lett. 14, 225-230 (2013).

5. Pors, A., Albrektsen, O., Radko, I. P. \& Bozhevolnyi, S. I. Gap plasmon-based metasurfaces for total control of reflected light. Sci. Rep. 3, 2155 (2013).

6. Barnes, W. L., Dereux, A. \& Ebbesen, T. W. Surface plasmon subwavelength optics. Nature 424, 824-830 (2003).

7. Khurgin, J. B. \& Sun, G. Scaling of losses with size and wavelength in nanoplasmonics and metamaterials. Appl. Phys. Lett. 99, 211106 (2011).

8. Lu, X., Shi, J., Liu, R. \& Guan, C. Highly-dispersive electromagnetic induced transparency in planar symmetric metamaterials. Opt. Express 20, 17581-17590 (2012)

9. Huang, Y.-W. et al. Toroidal Lasing Spaser. Sci. Rep. 3, 1237 (2013).

10. Shafiei, F. et al. A subwavelength plasmonic metamolecule exhibiting magneticbased optical Fano resonance. Nat. Nano. 8, 95-99 (2013).

11. Luk'yanchuk, B. et al. The Fano resonance in plasmonic nanostructures and metamaterials. Nat. Mater. 9, 707-715 (2010).

12. Kaelberer, T., Fedotov, V. A., Papasimakis, N., Tsai, D. P. \& Zheludev, N. I. Toroidal dipolar response in a metamaterial. Science 330, 1510-1512 (2010).

13. Huang, Y.-W. et al. Design of plasmonic toroidal metamaterials at optical frequencies. Opt. Express 20, 1760-1768 (2012).

14. Savasta, S. et al. Nanopolaritons: Vacuum Rabi splitting with a single quantum dot in the center of a dimer nanoantenna. ACS Nano 4, 6369-6376 (2010).

15. Schlather, A. E., Large, N., Urban, A. S., Nordlander, P. \& Halas, N. J. Near-field mediated plexcitonic coupling and giant Rabi splitting in individual metallic dimers. Nano Lett. 13, 3281-3286 (2013).

16. Anker, J. N. et al. Biosensing with plasmonic nanosensors. Nat. Mater. 7, 442-453 (2008).

17. Liu, N. et al. Magnetic plasmon formation and propagation in artificial aromatic molecules. Nano Lett. 12, 364-369 (2011).

18. Ekmekci, E. et al. Frequency tunable terahertz metamaterials using broadside coupled split-ring resonators. Phys. Rev. B 83, 193103 (2011).

19. Powell, D. A., Lapine, M., Gorkunov, M. V., Shadrivov, I. V. \& Kivshar, Y. S. Metamaterial tuning by manipulation of near-field interaction. Phys. Rev. B 82, 155128 (2010).

20. Enkrich, C. et al. Magnetic metamaterials at telecommunication and visible frequencies. Phys. Rev. Lett. 95, 203901 (2005).

21. Dolling, G., Wegener, M., Schadle, A., Burger, S. \& Linden, S. Observation of magnetization waves in negative-index photonic metamaterials. Appl. Phys. Lett. 89, 231118-231113 (2006).

22. Keiser, G. R. et al. Decoupling crossover in asymmetric broadside coupled splitring resonators at terahertz frequencies. Phys. Rev. B 88, 024101 (2013).

23. Soukoulis, C. M. \& Wegener, M. Past achievements and future challenges in the development of three-dimensional photonic metamaterials. Nat. Photon $\mathbf{5}$, 523-530 (2011).

24. Moser, H. O. \& Rockstuhl, C. 3D THz metamaterials from micro/ nanomanufacturing. Laser \& Photon. Rev. 6, 219-244 (2012).

25. Kanté, B. et al. Symmetry breaking and optical negative index of closed nanorings. Nat. Commun. 3, 1180 (2012).

26. Sersic, I., Frimmer, M., Verhagen, E. \& Koenderink, A. F. Electric and magnetic dipole coupling in near-infrared split-ring metamaterial arrays. Phys. Rev. Lett. 103 (2009).

27. Feth, N. et al. Electromagnetic interaction of split-ring resonators: The role of separation and relative orientation. Opt. Express 18, 6545-6554 (2010).
28. Zeng, Y., Dineen, C. \& Moloney, J. V. Magnetic dipole moments in single and coupled split-ring resonators. Phys. Rev. B 81, 075116 (2010).

29. Valentine, J. et al. Three-dimensional optical metamaterial with a negative refractive index. Nature 455, 376-379 (2008).

30. Liu, N., Liu, H., Zhu, S. N. \& Giessen, H. Stereometamaterials. Nat. Photon. 3, 157-162 (2009).

31. Liu, N. et al. Three-dimensional photonic metamaterials at optical frequencies. Nat. Mater. 7, 31-37 (2008).

32. Tseng, M. L. et al. Fabrication of multilayer metamaterials by femtosecond laserinduced forward-transfer technique. Laser \& Photon. Rev. 6, 702-707 (2012).

33. Yen, T. J. et al. Terahertz magnetic response from artificial materials. Science 303, 1494-1496 (2004).

34. Gundogdu, T. F. et al. Experimental demonstration of negative magnetic permeability in the far-infrared frequency regime. Appl. Phys. Lett. 89, 084103 (2006).

35. Fan, K., Strikwerda, A. C., Zhang, X. \& Averitt, R. D. Three-dimensional broadband tunable terahertz metamaterials. Phys. Rev. B 87, 161104 (2013).

36. Wu, P. C. et al. Magnetic plasmon induced transparency in three-dimensional metamolecules. Nanophotonics 1, 131-138 (2012).

37. Wu, P. C. et al. Vertical split-ring resonator based nanoplasmonic sensor. Appl. Phys. Lett. 105, 033105 (2014).

38. Chen, W. T. et al. Optical magnetic response in three-dimensional metamaterial of upright plasmonic meta-molecules. Opt. Express 19, 12837-12842 (2011).

39. Kriegler, C. E., Rill, M. S., Linden, S. \& Wegener, M. Bianisotropic photonic metamaterials. IEEE J. Sel. Topics Quantum Electron 16, 367-375 (2010).

40. Marqués, R., Medina, F. \& Rafii-El-Idrissi, R. Role of bianisotropy in negative permeability and left-handed metamaterials. Phys. Rev. B 65, 144440 (2002).

41. Linden, S. et al. Magnetic response of metamaterials at 100 terahertz. Science 306, 1351-1353 (2004).

42. Prodan, E., Radloff, C., Halas, N. J. \& Nordlander, P. A hybridization model for the plasmon response of complex nanostructures. Science 302, 419-422 (2003).

43. Nordlander, P., Oubre, C., Prodan, E., Li, K. \& Stockman, M. I. Plasmon hybridization in nanoparticle dimers. Nano Lett. 4, 899-903 (2004).

44. Zhang, J. et al. Tailoring alphabetical metamaterials in optical frequency: Plasmonic coupling, dispersion, and sensing. ACS Nano 8, 3796-3806 (2014).

45. Jain, P. K., Eustis, S. \& El-Sayed, M. A. Plasmon coupling in nanorod assemblies: Optical absorption, discrete dipole approximation simulation, and exciton-coupling model. J. Phys. Chem. B 110, 18243-18253 (2006).

\section{Acknowledgments}

The authors acknowledge financial support from Ministry of Science and Technology, Taiwan (Grant Nos. 103-2745-M-002-004-ASP, 102-2911-I-002-505 and 103-2911-I-002-594) and Academia Sinica (Grant No. AS-103-TP-A06). They are also grateful to National Center for Theoretical Sciences, Taipei Office, Molecular Imaging Center of National Taiwan University, National Center for High-Performance Computing, Taiwan, and Research Center for Applied Sciences, Academia Sinica, Taiwan for their support. G. Sun acknowledges support from AFOSR (Grant No. FA9550-14-1-0196, Dr. Gernot Pomrenke, Program Manager) and from AOARD (Grant No. AOARD-14-4073, Dr. Kenneth Caster, Program Manager). N. I. Zheludev also acknowledges sponsorship of: Science and Engineering research Council, UK and Ministry of Education of Singapore (Grant No. MOE2011-T3-1-005).

\section{Author contributions}

P.C.W. and W.L.H. designed and performed the experiments and data analysis, and wrote the manuscript; W.T.C., Y.-W.H. and C.Y.L. performed the experiments and data analysis; A.Q.L. and N.I.Z. discussed the experiments and prepared manuscripts; G.S. developed the theoretical aspects and wrote the manuscript; D.P.T. designed experiments, analyzed the results, and prepare the manuscripts. All authors discussed the results and commented on the manuscript.

\section{Additional information}

Competing financial interests: The authors declare no competing financial interests.

How to cite this article: Wu, P.C. et al. Plasmon coupling in vertical split-ring resonator metamolecules. Sci. Rep. 5, 9726; DOI:10.1038/srep09726 (2015).

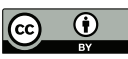

This work is licensed under a Creative Commons Attribution 4.0 International License. The images or other third party material in this article are included in the article's Creative Commons license, unless indicated otherwise in the credit line; if the material is not included under the Creative Commons license, users will need to obtain permission from the license holder in order to reproduce the material. To view a copy of this license, visit http://creativecommons.org/licenses/by/4.0/ 